\title{
A clampless and sutureless aortic anastomosis technique using an endograft connector for aortoiliac occlusive disease in which the aorta cannot be clamped or sewn due to calcification or scarring
}

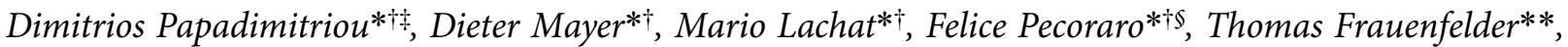 \\ Thomas Pfammatter**, Hideki Ueda*t†, Konstantinos Donas ${ }^{\ddagger}$, Frank J Veith*iss*** and Zoran Rancic*
}

Bypass surgery in aortoiliac or aortofemoral occlusive disease can be technically demanding and hazardous due to huge calcifications and/or patient co-morbidities. We report about mid-term results of a telescoping sutureless aortic anastomosis technique using endografts as connectors to address such challenging situations. This is a single-center experience (2004-2011) in seven patients $(63 \pm 6$ years) requiring aortoiliac (three) or aortofemoral (four) bypass surgery. In six cases, an aortic stent graft was telescoped into the infrarenal aorta and partly deployed within the aorta and partly outside the aorta. In the first case, a bifurcated stent graft was deployed and the iliac legs were prolonged extra-anatomically with surgical grafts to reach the femoral bifurcation. In the following five cases, a tapered tubular stent graft was deployed through the aortic wall, landing inside a bifurcated surgical graft that was extra-anatomically connected to the iliac or femoral arteries. In the last case, which presented a hostile abdomen and high-risk for extensive surgery, a similar technique was used, but on the iliac artery level. In that case, an iliac stent graft re-loaded 'upside down' was deployed through the left common iliac wall, landing distally inside a hand-made $10 \times 10 \mathrm{~mm}$ bifurcated surgical graft that was extra-anatomically connected to the left external iliac artery and to the right femoral artery. The distal anastomoses on the seven cases were performed either with running sutures (ten) or with VORTEC (four). Telescoping aortic and/or iliac anastomosis was successful in all patients. There was no perioperative mortality. One patient developed postoperative hyperperfusion of the left leg and necessitated fasciotomy. During a mean follow-up of $1.8 \pm 2$ years (minimum: 270 days, maximum: 7.1 years), all of the grafts remained patent and there was neither stent-graft migration nor stenosis on the level of the aortic or iliofemoral connection. One patient showed disease progression and required percutaneous transluminal angioplasty on the external iliac artery during follow-up. The uneventful perioperative course in these seven patients, with a follow-up of up to six years, underscores that this new technique can be considered in patients with aortoiliac or aortofemoral occlusive disease and in whom clamping and/or anastomosis is expected to be cumbersome or impossible.

Key words: aortoiliac occlusive disease; calcified aorta; cross-clamping; clampless-telescoping anastomosis; mid-term results

Accepted November 30, 2011

*Clinic for Cardiovascular Surgery; ${ }^{\dagger}$ University Hospital of Zurich, Zurich, Switzerland; ${ }^{\ddagger}$ Aristotle University of Thessaloniki, Hippocrateio Hospital, Thessaloniki, Greece; ${ }^{\mathfrak{S}}$ Vascular Surgery Unit, University of Palermo, Palermo, Italy; **Diagnostic Radiology, University Hospital of Zurich, Zurich, Switzerland; ${ }^{\dagger \dagger}$ Department of Cardiac Surgery and Vascular Surgery, KKR Sapporo Medical Center, Sapporo, Japan; ${ }^{\sharp}$ Cardiovascular Clinic, St Fransiscus Hospital, Münster, Germany; ${ }^{s \varsigma}$ The Cleveland Clinic, Cleveland, OH; ${ }^{* *}$ New York University Medical Center, New York, NY, USA

Correspondence to: Dimitrios N Papadimitriou, 5th Surgical Clinic, Hippocrateio Genaral Hospital and 424 Military General Hospital, Vascular and Endovascular Department, 54646 Thessaloniki, Greece; email: dimitrios.papadimitriou2@gmail.com

\section{Introduction}

Over the last decades, the management of aortoiliac occlusive disease has been marked by a series of debates over new approaches or concepts that have been introduced. Deciding which aortoiliac procedure is best for which patient is based on the general condition of the patient, the extent and distribution of the occlusive disease and the experience and training of the surgeon. Even if aortoiliac reconstruction, usually using prosthetic bypass grafts, offers the most successful durable results, ${ }^{1,2}$ patients might be referred to surgery when the disease is already severe and/or when percutaneous 
transluminal angioplasty (PTA) failed. Among these cases, there are some challenging aortic conditions of such severe calcification, that aortic cross-clamping and proximal anastomosis procedures might be risky and problematic. For these cases of challenging surgical aortic accessibility and manipulation, we developed a stent-graft-based connection tool for performing sutureless and clampless aortic or iliac anastomosis that had been shown to be safe (TransAtlantic Society Consensus [TASC]) to perform ${ }^{3}$ in a couple of cases. In this report, a more consistent experience with mid-term results is presented.

\section{Methods}

This is a single-center experience over a six-year period (December 2004-April 2011). Overall, seven consecutive patients (age $63 \pm 6$ years) were treated, mostly in the last two years, using a sutureless telescopic anastomotic technique (STAT) to overcome severe aortoiliac or aortofemoral occlusive disease. Indication for surgery included chronic and severe claudication or ischemic rest pain (TASC grade $\mathrm{C}$ ), and in one patient, toe gangrene was also present (TASC grade D). Indication for the STAT procedure was driven by severe co-morbidity and a hostile abdomen (in three patients) after prior abdominal surgery (Table 1) and the presence of extensive aortic and iliac or femoral calcifications.

Thoracoabdominal computerized tomography angiography (CTA), extending to the inguinal region, was performed in all patients in order to assess disease severity and

Table 1 Patient demographics and co-morbidities

\begin{tabular}{ll}
\hline Male/female & $6 / 1$ \\
Age (years) [mean years \pm SD] & $63 \pm 6$ \\
Hypertension & $6(85.7 \%)$ \\
Coronary artery disease & $3(42.86 \%)$ \\
COPD & $4(57.1 \%)$ \\
Diabetes mellitus & $1(14.28 \%)$ \\
Smoking & $7(100 \%)$ \\
Renal failure & $1(14.28 \%)$ \\
Dyslipidemia & $5(71.42 \%)$ \\
Hostile abdomen & $3(42.86 \%)$ \\
Obesity & $1(14.28 \%)$ \\
ASA classification & $\mathrm{III}$ \\
Other co-morbidities & $4(57.1 \%)$ \\
\hline
\end{tabular}

COPD, chronic obstructive pulmonary disease; ASA, American Society of Anesthesiologists

${ }^{*}$ One patient had thrombocytosis and anemia; one patient had hepatitis $\mathrm{B}$ virus, hepatitis $\mathrm{C}$ virus, hepatitis $\mathrm{A}$ virus, hyperthyroidism and chololithiasis; one patient had Hashimoto thyroiditis; and one patient had sleep apnea syndrome, hemorrhagic gastritis and duodenum valvulitis extension and to plan the procedure in detail. In all patients in this series, the aorta and both iliofemoral axes were severely diseased. Pathologies for treatment included complete infrarenal aortic occlusion (one), unilateral iliac occlusion (four) and bilateral iliac occlusion (one). In the first case, STAT was performed as a bailout procedure, after the intended end-to-end aortic anastomosis had failed. In the following six patients, the STAT procedure was performed according to preoperative planning.

\section{Surgical technique}

All procedures were performed in an operating room equipped with a mobile fluoroscopy machine. Open access to the aorta or iliofemoral vessels was achieved through a laparotomy (five) or retroperitoneal approach (two), with the patient under general anesthesia. After systemic heparinization (100 units heparin/kg bodyweight), the aortic wall was punctured at the site that was identified on CTA to be adequate for puncture. Moreover, digital control was performed before puncturing the aorta. This was followed by introduction of a stiff guide wire. The stent-graft delivery system was introduced through a standard bifurcated polyester graft $(n=6$, mostly $14 \mathrm{~mm} \times 7 \mathrm{~mm}$ or $16 \mathrm{~mm} \times 8 \mathrm{~mm}$ ) or a standard $10 \mathrm{~mm}$ expanded polytetrafluoroethylene (ePTFE) vascular graft $(n=1)$ and partially telescoped over the wire inside the aorta (Figure 1). Before deployment, the stent-graft position inside the aorta was controlled by fluoroscopy and the length to land into the surgical graft, at least for $2.5 \mathrm{~cm}$, was checked visually. The polyester graft was then advanced over the stent-graft delivery system close to the aortic wall and the stent graft deployed. Finally, the polyester graft and the stent graft were fixed (this is the fixing technique precisely) to the aorta with four Prolene sutures, running over and over the polyester graft, the stent graft and the aortic wall. In cases where some bleeding (three) was observed after stent-graft deployment (two), additional stitches through the graft and aortic wall were used. Finally, full stent-graft deployment was achieved using a Reliant balloon (Medtronic, World Medical Manufacturing Corp., Sunrise, FL, USA) inflated within the stent grafts. In all cases, after balloon inflation, any bleeding stopped. Distal anastomoses on the iliac $(n=7)$ or femoral arteries $(n=7)$ were performed using standard running suture technique (ten) or the Viabahn Open Rebranching TECnique (VORTEC) (four). ${ }^{4}$ Adjunctive outflow procedures were performed in two patients: eversion endarterectomy of the external iliac artery (one) and femoral bifurcation plasty (one). The first case we performed in 2004 as a bailout procedure was addressed in another way. 

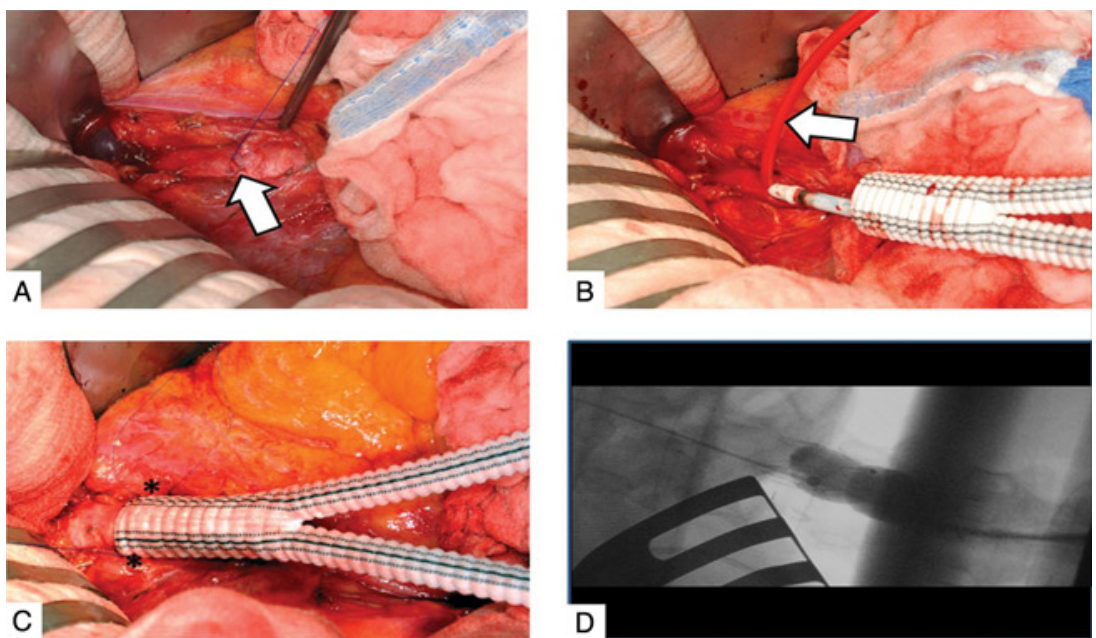

Figure 1 (a) Aortic wall, free of disease, is punctured and a stiff wire is passed inside the aorta. (b) Endograft connector (stent graft) is passed through a bifurcated Dacron graft and telescoped inside the aorta. (c) After stent-graft deployment, two sutures were used to stabilize the graft on the aorta. (d) Balloon (Reliant; World Medical Manufacturing Corp., Medtronic, Sunrise, FL, USA) dilation of the endograft connector, which is located half inside the aorta and half inside the bifurcated surgical graft, is performed to achieve full stent-graft expansion and complete sealing

Table 2 Types and number of endovascular and surgical grafts needed to perform the telescoping technique (also type and place of distal anastomosis seen)

\begin{tabular}{|c|c|c|c|c|}
\hline Case & Aortic stent grafts & Surgical graft & Distal anastomosis left & Distal anastomosis right \\
\hline 1 & Excluder $23 \mathrm{~mm} \times 12 \mathrm{~mm} \times 16 \mathrm{~mm}$ & $9 \mathrm{~mm}$ Dacron & CFA (E/S) & CFA (E/S) \\
\hline 2 & E-vita $16-20 \times 5 \mathrm{~cm}$ & $16 \times 8$ Dacron & EIA (E/S) & EIA (E/S with VORTEC) \\
\hline 3 & E-vita $16-20 \times 5 \mathrm{~cm}$ & $18 \times 8$ Dacron & DFA $(E / S)$ & DFA $(E / S)$ \\
\hline 4 & E-vita $16-16 \times 5 \mathrm{~cm}$ & $14 \times 7$ Dacron & EIA (E/S with VORTEC) & EIA (E/S) \\
\hline 5 & E-vita $16-16 \times 5 \mathrm{~cm}$ & $14 \times 7$ Dacron & EIA (E/S with VORTEC) & CIA (E/S with VORTEC) \\
\hline 6 & E-vita $16 \times 20 \times 5 \mathrm{~cm}$ & $14 \times 7$ Dacron & CFA (E/S) & DFA $(\mathrm{E} / \mathrm{S})$ \\
\hline 7 & Zenith iliac $20 \mathrm{~mm}-14 \mathrm{~mm} \times 10 \mathrm{~mm}$ & $10 \mathrm{~mm} \times 10 \mathrm{~mm}$ ePFTE (crossover) & EIA $(S, E / S)$ & CFA (E/S) \\
\hline
\end{tabular}

CIA, common iliac artery; EIA, external iliac artery; CFA, common femoral artery; DFA, deep femoral artery; E/E, end-to-end anastomosis; E/S, end-to-side anastomosis; ePTFE, expanded polytetrafluoroethylene

In this case, the abdominal aorta could not be crossclamped, not even tangentially. Therefore, to achieve bleeding control, a balloon catheter was introduced through the aorta and inflated intrathoracally (15 minutes). The aorta was then opened infrarenally (approximately $3 \mathrm{~cm}$ below the renal arteries) and thromboendarterectomy was performed. The result was unsatisfying and therefore it was decided to avoid suturing the highly destabilized aortic wall and to deploy a bifurcated stent graft (Excluder, WL Gore and Associates, Flagstaff, AZ, USA) infrarenally. Both limbs where extended with end-to-end sutures to $9 \mathrm{~mm}$ polyester grafts to reach the femoral bifurcations, where they were sutured in a standard fashion. The last case performed in this series, which presented severe co-morbidities and a high risk for transperitoneal surgery, was addressed with a limited retroperitoneal approach to be able to puncture the left common iliac artery in its distal part. A Zenith (Cook Medical, Bloomington, IN, USA) tapered iliac stent graft was first inversely reloaded to its shaft before being introduced through the iliac artery and landing into the distal infrarenal aorta. The distal end of that stent graft landed into a handmade bifurcated $10 \mathrm{~mm} \times 10 \mathrm{~mm}$ ePTFE surgical graft that was sutured to the left distal external iliac artery and the right common femoral artery. The distal anastomoses on the seven cases were performed either with running sutures (ten) or VORTEC (four). Technical details to perform VORTEC are described elsewhere. ${ }^{5}$ Once all anastomosis had been completed, heparin was fully reverted with protamine. At the end of the procedure, pressure measurements on the graft and Doppler control were performed on the distal outflow vessels. In all cases, normal pressures and flow pattern were registered. Periopreative anticoagulation consisted of low-dose heparinization and dual antiplatelet therapy with $100 \mathrm{mg}$ aspirin and $75 \mathrm{mg}$ clopidogrel (Table 2).

\section{Postoperative follow-up}

Postoperatively and during mid-term follow-up, patency was determined by Doppler and ankle brachial index (ABI) 
measurements and CTA, prior to discharge and at one month, six months and one year postoperatively (Figure 2). significantly from $0.48 \pm 0.12$ to $0.86 \pm 0.26 \quad(P<0.001)$, followed by clinical status improvement in all patients (Table 3).

\section{Discussion}

A variety of operative approaches and methods are available for the management of aortoiliac disease. Direct bilateral aortoiliac or aortofemoral reconstruction using prosthetic bypass grafts offers the best long-term results ${ }^{2,6}$ Aorto bi-femoral bypass is the most frequently performed reconstructive procedure for aortoiliac occlusive disease and offers the most effective and durable method of revascularization available. ${ }^{7}$ This technique is considered the 'gold standard' of treatment, with the best long-term patency rates of any other lower extremity bypass (85-90\%) in five years. $^{7,8}$ Operative mortality for revascularization can be as low as about $5 \%{ }^{1,7}$ But in some cases, surgery has to face some quite challenging aspects, such as accessibility to the aorta, especially in scar tissue, aortic cross-clamping on a calcified wall or difficulty of making a proximal or distal anastomosis in a calcified and heavily diseased artery. Huge atherosclerotic plaques in the pararenal aortic segment require supraceliac or even thoracic cross-clamping through

Table 3 Operative statistics and postoperative follow-up data

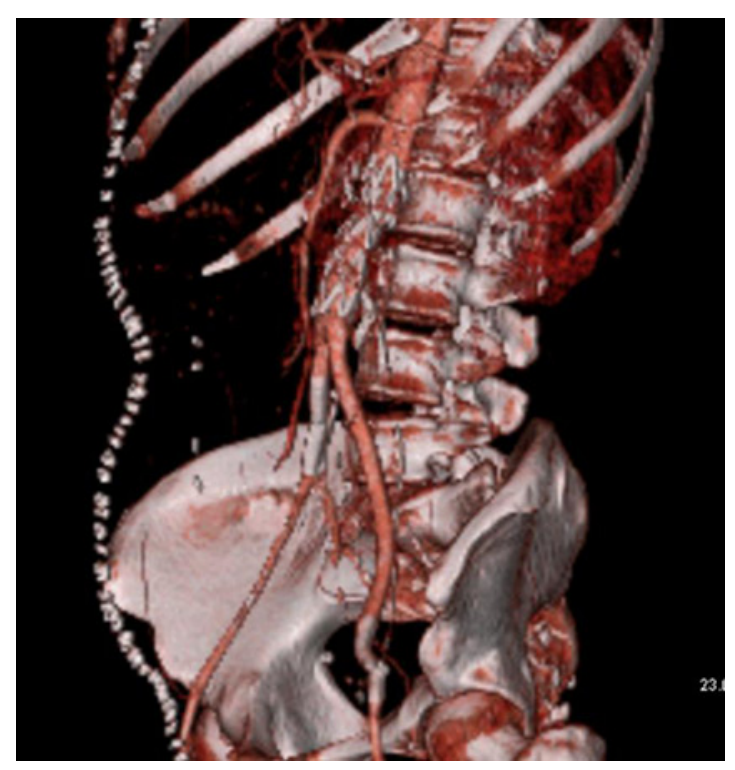

Figure 2 Postoperative CTA showing the endograft connector (iliac limb, E-Vita, JOTEC GmbH, Hechingen, Germany) landing inside a bifurcated surgical graft (Intergard Silver, InterVascular, MAQUET GmbH Co KG, Rastatt, Germany) which reached distally the right common iliac artery and on the left side the distal external iliac artery. Note that both distal connections have been performed with VORTEC. CTA, computerized tomography angiography

\begin{tabular}{ll}
\hline Type of incision & 5 \\
Medial laparotomy & 2 \\
Retroperitoneal approach & $294( \pm 63)$ \\
Duration of intervention (min) & $1(12)$ \\
Aortic occlusion balloon (min) & \\
Type of anesthesia & $7(100 \%)$ \\
$\quad$ General & $2.4( \pm 3.1)$ in 3 cases \\
Blood transfusion (RBC units) & $4(57.1 \%)$ \\
No. of patients in ICU & $1.1( \pm 0.4)$ \\
ICU stay (days) & 5 \\
Additional operative procedures & 1 \\
Endarterectomy of distal aorta & 1 \\
Eversion endarterectomy of EIA & 1 \\
PTA CFA & 1 \\
Femoral bifurcation plasty & 1 \\
Profundaplasty & 0 \\
30-day mortality & $100 \%$ \\
Technical success & \\
Postoperative complications & 0 \\
Procedure-related & $1(14.28 \%)$ \\
Reinterventions (distal angioplasty) & $14( \pm 24)$ \\
Follow-up time (months) & \\
\hline
\end{tabular}

RBC, red blood cell; ICU, intensive care unit; EIA, external iliac artery; PTA, percutaneous transluminal angioplasty; CFA, common femoral artery 
a thoracoabdominal approach that increases perioperative complication rate and mortality rate (12.4\%) significantly. ${ }^{9,10}$ Severe periaortic inflammation may cause similar problems. Trying to cross-clamp a severely diseased aorta can induce plaque embolization, extensive aortic wall disruption and uncontrollable bleeding. ${ }^{11}$ Moreover, performing an anastomosis in inadequate aortic tissue quality bears the risk of late pseudoaneurysm, aortic tearing or dissection. As an alternative, intra-aortic occlusion with a balloon has been described. Unfortunately, even such balloons can lead to distal debris embolization and aortic wall rupture. ${ }^{12,13}$

Telescoping stent-graft devices into the aorta, as was successfully performed in our cases, is another surgical option that could be considered in challenging open surgery cases. With this technique, aortic minimal vessel preparation is sufficient to perform aortic anastomosis. In our first case, an intra-aortic balloon catheter was used to control the inflow, while the aorta was transected in order to perform endarterectomy and conventional aortic-to-graft end-to-end sutured anastomosis. As this failed, a bifurcated stent graft was introduced under visual control into the transected aorta and deployed infrarenally. Based on this experience, it became obvious that the aortic stent-graft introduction and deployment could be performed by puncturing a soft aortic area, just above the aortic occlusion level. As a consequence, the following cases were performed by puncturing the aortic wall and the stent grafts telescoped into the aorta using the Seldinger technique. Moreover, based on recent advances with the VORTEC, and similar to that technique, aortic stent grafts were deployed partially within the aorta and partially inside a surgical graft, preferably a bifurcated polyester fabric in all following cases. Distal anastomosis has been performed either with running sutures or to overcome accessibility or continuous calcified segments by using the VORTEC. The advantage of the Viabahn device (WL Gore and Associates) is that it is not elongated in the delivery system, so there is no foreshortening in deployment, which makes accurate length positioning of the endograft more straightforward. Moreover, one-year patency rates are superior to bare stent in this type of location (100 versus $92.5 \%, P=0.037){ }^{14,15}$ There are also some technical details and considerations that should be pointed out in order to achieve a satisfactory stent-graft anchorage and sealing after deployment. A balloon dilation and four additional sutures, from aortic wall to stent graft at entry point and backwards, should be performed in order to secure the connection site and the stability of the graft. Following the emerging experience of the technique in these six years and the satisfactory results, it is recommended to land the distal end of the stent graft within a surgical graft, as performed in all cases but the first one. This concerns the long-term durability of the stent graft fabric and the perigraft tissue reaction. This is because potential fabric failure of an unprotected stent graft would be prone to bleeding complications than one protected inside a surgical graft. Also, in case of stent fracture, the struts might perforate the bowel, causing a major complication. Based on this, it should be pointed out that there is no need for a strong follow-up CTA in these patients. The potential complications are not endoleak or sac enlargment as in aneurysms, but migration, fraction or fabric corruptions that are not considered to be early complications for an endovascular graft. So, for the follow-up of these cases, only ABI measurement is required and a CTA is performed postoperatively and yearly thereafter. Bearing in mind the operating details, we should be focused on the shorter operation time to complete the procedure, less blood units needed to be transfused, reduced ICU and hospital stay and significantly less complication rates - periprocedural and mid-term - compared with difficult cases of severe and extensive aortoiliac occlusive disease. ${ }^{16}$ Also, this technique can be performed both by a transperitoneal and retroperitoneal approach, making it more attractive in the hostile abdomen or for patients prepared for future bowel operation. Where the femoral region is free of disease, there are still opportunities for future endovascular or open procedures when an aortoiliac bypass is performed using the telescopic technique.

\section{Conclusions}

The clampless and sutureless aortic anastomosis technique using an endograft connector for aortoiliac occlusive disease is a less invasive revascularization technique that shows very supportive mid-term results. Extended follow-up with longterm results and larger number of patients and probably a comparative study will be mandatory before it can be concluded that this endograft-based anastomotic technique is superior to other surgical strategies. Nevertheless, this technique could be a helpful tool for endoscopic or robotic aortoiliac revascularization.

\section{Declarations}

\section{Conflicts of interest: None}

\section{References}

1 McDaniel M, Macdonald P, Haver R, et al. Published results of surgery for aortoiliac occlusive disease. Ann Vasc Surg 1997;11: 425-42 
2 de Vries SO, Huning MG. Results of aortic bifurcation grafts for aortoiliac occlusive disease: a meta-analysis. J Vasc Surg 1997; 26:558-69

3 Donas K, Rancic Z, Frauenfelder T, et al. Sutureless telescoping aortic anastomotic technique for hybrid surgical treatment of aortoiliac occlusive disease. J Endovasc Ther 2010;17: 251-4

4 Lachat M, Mayer D, Criado FJ, et al. New technique to fascilitate renal revascularization with use of telescoping self-expanding stent grafts: VORTEC. Vascular 2008;16:69-72

5 Rancic Z, Mayer D, Pfammater $\mathrm{T}$, et al. A new sutureless telescoping anastomotic technique for major aortic branch revascularization with minimal dissection and ischemia. Ann Surg 2010;252:884-9

6 Brewster D. Clinical and anatomical considerations for surgery in aortoiliac disease and results of surgical treatment. Circulation 1991;82:I42-I51

7 Nulcare RJ, Royster TS, Lynn RA, et al. Long term results of operative therapy for aortoiliac disease. Arch Surg 1978;113:601-4

8 Rutherford RB. Options in the surgical management of aortoiliac occlusive disease: a changing prospective. Cardiovasc Surg 1999; $7: 5-12$

9 Stanley JC, Criado E, Eliason JL, et al. Abdominal aortic coarctation: surgical treatment of 53 patients with a thoracoabdominal bypass, patch aortoplasty or interposition aortoaortic graft. J Vasc Surg 2008;48:1073-82
10 Schneider JR, Gottner RJ, Golan JF. Supraceliac versus infrarenal aortic cross-clamp for repair of non-ruptured infrarenal and juxtarenal abdominal aortic aneurysm. Cardiovasc Surg 1997; 5:279-85

11 Babin-Ebell J, Gimpel-Henning K, Sievers HH, Scharfschwerdt M. Influence of clamp duration and pressure on endothelial damage in aortic cross-clamping. Interact CardioVasc Thorac Surg 2010; 10:168-71

12 Matsuda H, Tanaka Y, Hino Y, et al. Transbrachial arterial insertion of aortic occlusion balloon catheter in patients with shock from ruptured abdominal aortic aneurysm. J Vasc Surg 2003;38:1293-6

13 Tang X, Guo W, Yang R, et al. Use of aortic balloon occlusion to decrease blood loss during sacral tumor resection. J Bone Joint Surg 2010;92:1747-53

14 Ashan A, Gregory Modrall J, Lopez J, et al. Emerging role of endovascular grafts in complex aortoiliac occlusive disease. $J$ Vasc Surg 2003;38:486-91

15 Ricci MA, Morris CS, Forgione P, et al. Primary endovascular grafting of iliac occlusive disease is superior to stent alone-initial results from randomized, multicenter trial. Presented at the Forty-ninth Scientific Meeting for the American Association for Vascular Surgery, Baltimore, MD, 12-13 June 2001

16 Crawford ES, Bomberger RA, Glaeaser DH, et al. Aortoiliac occlusive disease: factors influencing survival and function following reconstructive operation over a twenty-five-year period. Surgery 1981;90:1055-67 